\title{
Are dietary diversity scores related to the socio-economic and anthropometric status of women living in an urban area in Burkina Faso?
}

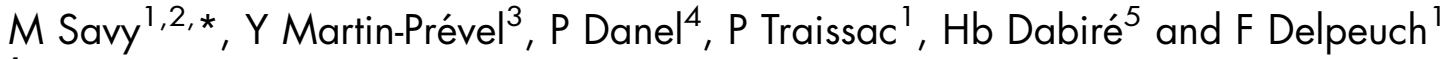 \\ 'Research Unit 106 'Nutrition, Food, Societies' (WHO Collaborating Centre for Nutrition), Institut de Recherche \\ pour le Développement (IRD), BP 64501, F-34394 Montpellier Cedex 5, France: ${ }^{2}$ Doctoral School 393 'Public \\ Health: Epidemiology and Biomedical Information Sciences', Université Pierre et Marie Curie, Paris, France: \\ ${ }^{3}$ Research Unit 106 'Nutrition, Food, Societies', IRD, Ouagadougou, Burkina Faso: ${ }^{4}$ Sciences and Technology of \\ Biology, Nutrition and Human Food, National Institute of Agronomy of Paris-Grignon, Paris, France: ${ }^{5}$ Institut \\ Supérieur des Sciences de la Population (ISSP), Ouagadougou, Burkina Faso
}

\section{Submitted 29 August 2006: Accepted 8 March 2007: First published online 13 June 2007}

\begin{abstract}
Objectives: To study dietary diversity and its relationship with socio-economic and nutritional characteristics of women in an urban Sahelian context.

Design: A qualitative dietary recall was performed over a 24-h period. Dietary diversity scores (DDS = number of food groups consumed) were calculated from a list of nine food groups (DDS-9) or from a list of 22 food groups (DDS-22) which detailed both micronutrient- and energy-dense foods more extensively. Body mass index (BMI), mid upper-arm circumference and body fat percentage were used to assess the nutritional status of the women.

Setting and subjects: Five hundred and fifty-seven women randomly selected in two districts of Ouagadougou, the capital of Burkina Faso.

Results: The mean DDS-9 and DDS-22 were $4.9 \pm 1.0$ and $6.5 \pm 1.8$ food groups, respectively. In the high tertile of DDS-22, more women consumed fatty and sweetened foods, fresh fish, non-fatty meat and vitamin-A-rich fruits and vegetables. The DDS-9 was not associated with the women's socio-economic characteristics whereas the DDS-22 was higher when the women were younger, richer and had received at least a minimum education. Mean BMI of the women was $24.2 \pm 4.9 \mathrm{~kg} \mathrm{~m}^{-2}$ and $37 \%$ of them were overweight or obese $\left(\mathrm{BMI} \geq 25 \mathrm{~kg} \mathrm{~m}^{-2}\right)$. Neither the DDS-9 nor the DDS-22 was associated with the women's anthropometric status, even though there was a trend towards fewer overweight women in the lowest tertile of DDS-22.

Conclusion: In this urban area, the qualitative measurement of dietary diversity is not sufficient to identify women at risk of under- or overweight.
\end{abstract}

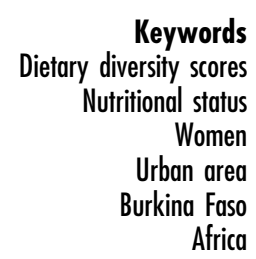

As no single food contains all necessary nutrients, diversity in dietary sources is needed to ensure a balanced and healthy diet. It is well documented in developed countries that dietary diversity at different ages, and represented by different types of scores, is strongly associated with nutrient adequacy and is thus an essential element of diet quality ${ }^{1-5}$. There is less evidence showing such associations in developing countries, but the few studies that are available provide support for the assumption that dietary diversity and variety scores are also good proxies of the nutrient adequacy of the diet in poor countries ${ }^{6-9}$. Moreover, it has been reported that these scores can be useful indicators of household food security ${ }^{10}$ and that they are positively associated with the nutritional status of children ${ }^{11-15}$. In previous studies in a rural area in Burkina Faso, we recently demonstrated that such dietary diversity scores calculated over a 24-h period were also inversely associated with underweight in adults ${ }^{16-18}$.

Thus, at least in developing countries, dietary diversity is now more and more frequently assessed using simple tools such as the dietary diversity score (DDS), defined as the number of food groups, subgroups or food items consumed over a reference period. Such scores are now used by international institutions like ORC Macro in their Demographic and Health Survey (DHS), in the FANTA (Food and Nutrition Technical Assistance) Project and by the International Policy Research Institute, but in most cases these scores are seen as proxies of adequate/poor 
micronutrient intakes. Yet our previous work in rural Burkina Faso showed that higher DDS was associated with more frequent consumption of both micronutrientand energy-rich foods ${ }^{17}$. In such an underprivileged context, dietary diversity could be considered as equivalent to overall diet quality since nutritional issues were essentially macro- and micronutrient deficiencies. Nevertheless, as soon as people have access to a range of different foods, diet quality cannot be restricted to adequate micronutrient intakes but must also take into account moderation behaviours regarding intakes in energy, saturated fat, cholesterol, sodium and sugar. This is true not only for developed countries but also for developing countries, particularly in urban areas where undernutrition and food insecurity coexist with dietary excess and obesity, sometimes within the same household $^{19-21}$. Consequently, some authors have proposed diet quality indices that take this dual risk into consideration. Most of these indices are based on US nutritional recommendations and aim at identifying individuals who both succeed in meeting their nutrient needs and in reducing their consumption of 'unhealthy' foods such as refined sugar or saturated fats ${ }^{22-25}$. However, these indices were developed for industrialised countries and require the quantitative assessment of nutrient and/or food groups, which is more difficult to achieve in developing countries where the level of education is generally low and where populations often share food from a communal bowl ${ }^{26}$.

The aims of the present study were to measure dietary diversity in women living in the capital city of Burkina Faso through simple scores and to study the relationships between these DDS and the women's socio-economic and anthropometric characteristics. We explored whether such simple scores measured in an urban African context could reflect the dual risk described above. Therefore we used both a DDS based on a list of nine food groups that we had already used in rural areas, with another DDS based on a list of 22 food groups which better represented both micronutrient- and energy-rich foods.

\section{Methods}

\section{Study area and sampling}

The study was conducted in Ouagadougou, the capital of Burkina Faso. The city covers an area of $220 \mathrm{~km}^{2}$, divided into districts, with amenities in the town centre and peripheral districts without amenities. According to the most recent population census in 1996, Ouagadougou had about 750000 inhabitants but the population is now estimated at 1200000 inhabitants ${ }^{27}$. A cross-sectional domestic survey was carried out in May 2005 in two districts of Ouagadougou: Taabtenga, a poor district of about 3500 inhabitants with no amenities; and Wemtenga, a richer district of about 2500 inhabitants with amenities.
These districts were involved in the Demographic Monitoring System carried out by the Institut Supérieur des Sciences de la Population (ISSP) [Higher Institute of Population Sciences]. Most of the sociodemographic and economic information we needed was thus already available. Although we did not intend to use a strictly representative sample of the population of Ouagadougou, we compared the social and economic data of the two districts with the Ouagadougou sample used by the most recent $\mathrm{DHS}^{28}$. It turned out that, if we selected the same number of subjects in each district, the study sample correctly reflected the global social and economic situations observed in Ouagadougou. Given the explorative nature of the present study, we therefore decided to randomly select 300 women aged from 20 to 59 years in each district from the ISSP database, giving a total sample of 600 subjects.

\section{Dietary diversity scores}

Information on food consumption was collected by a qualitative dietary recall over the previous $24 \mathrm{~h}$. The women were asked to spontaneously recall all the dishes, sauces, snacks, drinks and other foods they had consumed the previous day. We then prompted them to be sure that no food had been forgotten. Next, the women were asked to describe all the ingredients in the food they had eaten. The ingredients were then coded by a fieldworker using a pre-defined list of 22 food groups. This information allowed us to construct dietary diversity scores, defined as the number of different food groups consumed over a 24 -h period. The first score was calculated using the list of 22 food groups in order to obtain (1) information about the consumption of micronutrientrich foods - cereals; roots/tubers; beans/pulses; vitaminA-rich fruits/vegetables; other fruits (including fresh fruit juices); other vegetables; non-fatty meat (beef, mutton, game, horsemeat) and poultry; liver; fresh fish; dried fish; milk/dairy products; and (2) consumption of energy-rich foods and/or unhealthy foods - nuts/seeds; fatty meat (pork, cooked meats); animal fats; vegetal fats; frying oil (used for fried foods); red palm oil; cheese; eggs; sugar/ sweetened products; sweetened drinks (sodas, traditional and industrial juices); alcohol. The codes for the items on the list of 22 food groups were then aggregated in the classification system proposed at a recent workshop on dietary diversity held in Rome ${ }^{29}$, which includes nine food groups: cereals/roots/tubers; pulses/nuts; vitamin-Arich fruits/vegetables; other vegetables; other fruits; meat/ poultry/fish; eggs; milk/dairy products; oils/fats. The second DDS was calculated from this new classification. Neither the frequency of consumption nor a minimal amount of food was taken into consideration. In the following, the DDS constructed from the list of nine food groups and the list of 22 food groups will be called respectively DDS-9 and DDS-22. Both DDS were used as 
quantitative variables and were also categorised into tertiles to distinguish diets of high, medium and low diversity.

\section{Antbropometric measurements}

Anthropometric measurements were taken in accordance with World Health Organization recommendations ${ }^{30}$. The height of the subjects was measured to the nearest $\mathrm{mm}$ with locally made portable devices equipped with height gauges (SECA 206 Bodymeter). Their weight was measured to the nearest $100 \mathrm{~g}$ on a foot-to-foot impedance analyser with a maximum weighing capacity of $130 \mathrm{~kg}$ (Bodymaster $^{\mathrm{TM}}$; SEB Group). Body mass index (BMI; weight divided by the square of height, $\mathrm{kg} \mathrm{m}^{-2}$ ) was then calculated. After the age, sex and the height of the subject were entered, the scales also gave the fat mass (in kg) by impedance measurements. The body fat percentage was calculated $(\mathrm{BFP}=($ fat mass/total weight $) \times 100)$ and then corrected for the black population using the following formula ${ }^{31}: \mathrm{BFP}_{\mathrm{C}}=2.1+1.04157 \times \mathrm{BFP}$. Finally, the mid upper-arm circumference (MUAC) was measured to the nearest $\mathrm{mm}$ using a non-stretchable measuring tape. Women who said they were pregnant $(n=26)$ and women with unreliable anthropometric measurements $(n=7)$ due to a physical handicap or other causes were excluded from analyses using anthropometrics.

\section{Otber information}

Two additional questionnaires were addressed to the women in order to collect economic and sanitary data at the household level, on the one hand, and data on sociodemographics, care level, dietary perceptions and habits (number, time and location of meals) at the individual level, on the other. Indices were constructed to summarise some of this information:

- A household economic index was constructed using a correspondence analysis performed on the matrix of indicator variables that coded for housing quality (type and size of housing, number of persons per room, quality of walls and roofs) and facilities (electricity, running water and origin of drinking water, nature of toilets and shower, waste evacuation), for assets (television, telephone, refrigerator, video tape recorder) and modes of transport. For a given household, the score on the axis of the first principal component of the correspondence analysis gives a coordinate that is interpreted as a summary indicator of its economic level $^{32}$. This index was then divided into tertiles.

- A household bygiene index was constructed from data about the source of drinking water, type and sharing of toilets, evacuation of rubbish and waste water, and a spot-check of the cleanliness of the compound. Positive or negative points were then attributed based on favourable or unfavourable situations. The index ranged from -3 to +4 and was divided into tertiles according to its distribution to identify low, medium and high hygienic conditions for the households.

- An individual care for women index assessed the level of attention and support given to women by the other members of the household. Using a similar point system, this index was constructed from the following information: power of decision (about personal and child health, family planning, household purchases, meals) and financial autonomy of the woman, verbal or physical ill-treatment and general consideration of the woman in her household. The index values ranged from -8 to +9 within the sample and the women were classified in low, medium and high 'care for women' according to the distribution of the index.

Concerning their perception of diet, the women were asked to judge whether their own diet was 'sufficient' or 'insufficient' in terms of quantity and 'rather varied' or 'not varied enough' in terms of quality.

All of this information had either already been collected by the ISSP or was collected by ourselves. The interviews were conducted by six local fieldworkers. Two other fieldworkers performed anthropometric measurements and checked the quality of the field data. All fieldworkers spoke French as well as the main local languages. The general supervision of the survey was ensured by the first three authors of this paper.

\section{Data management and statistical analyses}

Data entry was performed with EpiData software, version $3.1^{33}$. Data quality was ensured by quality checks at data entry, by double entry and also by further data cleaning. Data management, including computation of the DDS from the dietary recall, was performed with SAS system version $9.1^{34}$. To analyse the relationships between dietary diversity and socio-economic factors, the DDS were used as response variables and analysed as a function of these factors. Next, we analysed the relationship between the women's nutritional status and the DDS. For this analysis, the anthropometrics were used as response variables and analysed as a function of the DDS divided into tertiles. We then re-ran the same models after having included sociodemographic and economic factors which could play a role of confounding factors. The general linear model was used for quantitative response variables and the logistic model for categorical responses. Stratification of the sample according to the two districts was taken into account with specific procedures in SAS V9.1 (Proc Surveyreg and Proc Surveylogistic). The first type error rate was set at 0.05 for all analyses.

\section{Etbics}

The protocol of the study was approved by the Ethics Committee of the Ministry of Health of Burkina Faso. The 
objectives and principles of the study were explained to all participants in their own language. All the women gave oral consent to participate in the study.

\section{Results}

Initially, a sample size of 600 was envisaged but only 557 women had complete data on dietary diversity. Of these 557 women, 526 were eligible. Data on sociodemographic and economic conditions or anthropometric status were incomplete in the case of 45 eligible women, so the final sample comprised 481 eligible women with complete data.

\section{Characteristics of the sample}

The average age of the women in our sample was 37 years. Most were married or lived in cohabitation and were Moslem. Among them, 42\% had never attended school and a third was unemployed or inactive. Concerning anthropometric characteristics (Table 1), the mean BMI of the women was rather high $\left(24.2 \pm 4.9 \mathrm{~kg} \mathrm{~m}^{-2}\right)$. Among them, $37 \%$ were overweight $\left(\mathrm{BMI} \geq 25 \mathrm{~kg} \mathrm{~m}^{-2}\right)$ including $13 \%$ obese $\left(\mathrm{BMI} \geq 30 \mathrm{~kg} \mathrm{~m}^{-2}\right)$, and $8 \%$ were underweight $\left(\mathrm{BMI}<18.5 \mathrm{~kg} \mathrm{~m}^{-2}\right.$ ). Based on the body fat ranges for standard adults reported by Gallagher et $a l^{35}$, the mean $\mathrm{BFP}_{\mathrm{c}}$ was rather high.

\section{Dietary diversity}

The DDS-9 ranged from two to eight food groups with a mean of $4.9 \pm 1.0$ food groups, whereas the DDS-22 ranged from two to 13 food groups with a mean of $6.5 \pm 1.8$ (Fig. 1). The women's diet almost always included the group of cereals/roots/tubers - in practice essentially cereals - and the meat/fish group, mainly non-fatty meat, fresh and dried fish. Most often, the diet also included vegetables (other than vitamin-A-rich vegetables) and fats, especially vegetal fats and frying oil. Sometimes the women also consumed vitamin-A-rich fruits or vegetables, legumes (nuts/seeds and beans/

Table 1 Anthropometric characteristics of the sample $(n=481)$

\begin{tabular}{lc}
\hline & Mean \pm SD or \%* \\
\hline Height $(\mathrm{cm})$ & $162.9 \pm 5.9$ \\
Weight $(\mathrm{kg})$ & $64.4 \pm 14.4$ \\
BMI $\left(\mathrm{kg} \mathrm{m}^{-2}\right)$ & $24.2 \pm 4.9$ \\
Mid upper-arm circumference $(\mathrm{cm})$ & $28.8 \pm 4.3$ \\
Body fat percentage correctedt & $36.2 \pm 8.8$ \\
BMl category $(\%)$ & \\
$\quad$ Underweight $\left(\mathrm{BMl}<18.5 \mathrm{~kg} \mathrm{~m}^{-2}\right)$ & 8.0 \\
$\quad$ Normal $\left(18.5 \mathrm{~kg} \mathrm{~m}^{-2} \leq \mathrm{BMl}^{-2} 25 \mathrm{~kg} \mathrm{~m}^{-2}\right)$ & 55.4 \\
Overweight $\left(\mathrm{BMI} \geq 25 \mathrm{~kg} \mathrm{~m}^{-2}\right)$ & 36.6 \\
\hline
\end{tabular}

SD - standard deviation; BMI - body mass index.

* Mean \pm SD for continuous variables and percentage for categorical variables.

† Corrected for black population. pulses) and fruits not rich in vitamin A. Milk and dairy products (mainly milk and yoghurts) were less consumed. The consumption of eggs was very rare (Table 2 ).

In order to better understand the meaning of a high dietary diversity in this urban context, we detailed the frequency of consumption of food groups as a function of tertiles of the DDS based on the list of 22 food groups (Fig. 2). In the high tertile of the DDS-22, there were more women who consumed fatty products such as frying oil and vegetable fats, sweetened products such as sugar and sweetened drinks. However, there were also more women who consumed fresh fish, non-fatty meat, roots and tubers, and vitamin-A-rich fruits and vegetables. To a lesser extent, there were also more women who consumed beans and pulses, milk and yoghurts, nuts and seeds, and fruits and vegetables not rich in vitamin A. There were no differences between tertiles for the groups of cereals and dried fish, nor for alcohol, fatty meat, eggs, liver, cheese and red palm oil, which were very rarely consumed in any of the three categories of DDS-22.

Finally, we investigated the source of dietary diversity by looking at the total numbers of dishes, sauces, snacks and drinks consumed over the $24-\mathrm{h}$ period as a function of tertiles of the DDS-9 and DDS-22 (Table 3). We observed that the number of snacks consumed was much higher when both DDS were higher, and the number of drinks was higher when the DDS-22 was higher. To a lesser extent, the frequency of consumption of dishes
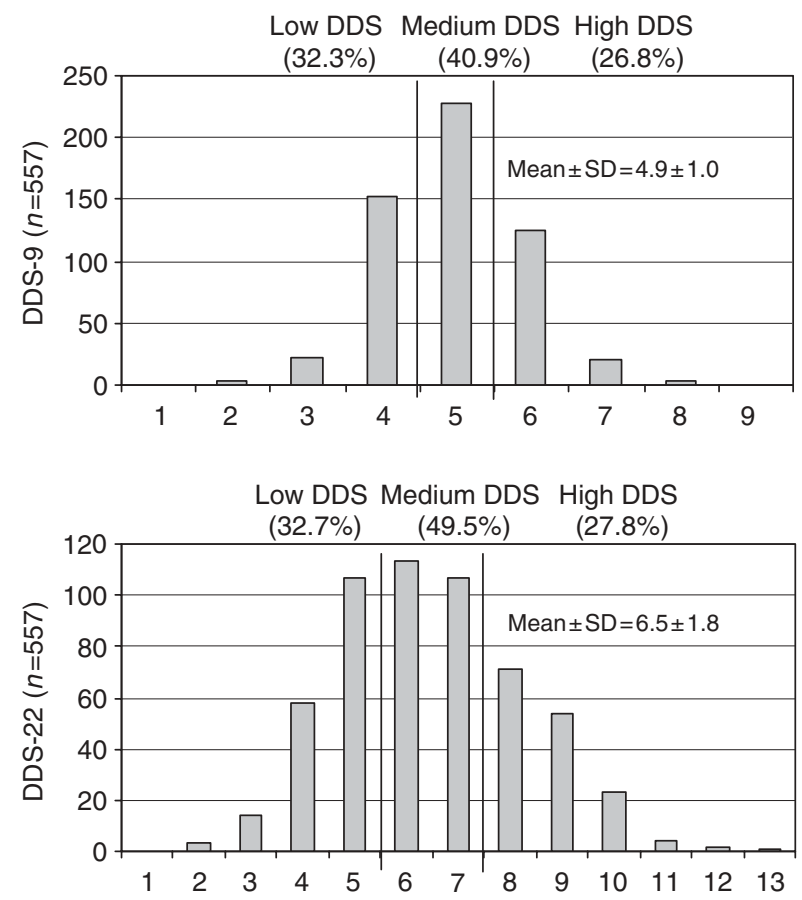

Fig. 1 Distribution and tertiles of the dietary diversity score (DDS) calculated from the lists of nine (DDS-9) and 22 (DDS22) food groups (SD - standard deviation) 
Table 2 Percentage of women who consumed food from the lists of nine and 22 food groups over the $24-\mathrm{h}$ period

\begin{tabular}{|c|c|c|}
\hline 9-food-group list & 22-food-group list & $\%$ \\
\hline \multirow[t]{3}{*}{ Cereals/roots/tubers } & - & 99.5 \\
\hline & Cereals & 98.7 \\
\hline & Roots/tubers & 10.8 \\
\hline \multirow[t]{3}{*}{ Legumes } & - & 36.3 \\
\hline & Nuts/seeds & 24.9 \\
\hline & Beans/pulses & 16.3 \\
\hline \multirow{2}{*}{$\begin{array}{l}\text { Vitamin-A-rich fruits and } \\
\text { vegetables }\end{array}$} & - & 49.0 \\
\hline & $\begin{array}{l}\text { Vitamin-A-rich fruits and } \\
\text { vegetables }\end{array}$ & 49.0 \\
\hline \multirow[t]{2}{*}{ Other vegetables } & - & 93.2 \\
\hline & Other vegetables & 93.2 \\
\hline \multirow[t]{2}{*}{ Other fruits } & - & 26.0 \\
\hline & Other fruits & 26.0 \\
\hline \multirow[t]{6}{*}{ Meat/fish } & - & 93.4 \\
\hline & Fatty meat & 0.9 \\
\hline & Non-fatty meat & 48.3 \\
\hline & Liver & 0.5 \\
\hline & Fresh fish & 35.4 \\
\hline & Dried fish & 31.4 \\
\hline \multirow[t]{2}{*}{ Eggs } & & 0.7 \\
\hline & Eggs & 0.7 \\
\hline \multirow[t]{3}{*}{ Milk and dairy products } & - & 10.8 \\
\hline & Milk/yoghurt & 10.6 \\
\hline & Cheese & 0.4 \\
\hline \multirow[t]{5}{*}{ Fats } & - & 84.4 \\
\hline & Vegetal fats & 76.1 \\
\hline & Animal fats & 1.4 \\
\hline & Red palm oil & 0.2 \\
\hline & Frying oil & 40.6 \\
\hline \multirow[t]{4}{*}{-} & - & \\
\hline & Sugar/sweet products & 44.7 \\
\hline & Sweetened drinks & 31.6 \\
\hline & Alcohol & 4.5 \\
\hline
\end{tabular}

was also higher when both DDS were higher, whereas the frequency of consumption of sauces was lower.

\section{Relationship between DDS and socio-economic characteristics}

The mean DDS-9 was not statistically associated with the socio-economic variables, except with the perception of the women about their diet in terms of quantity (Table 4). Indeed it was significantly higher for the women who rated their diet as sufficient $(P=0.01)$. In contrast, the mean DDS-22 was associated with several sociodemographic and economic variables. This score was higher when the women lived in the richer district (Wemtenga), when they were younger and when they had attended school, when they were single or married compared with widowed and divorced, and when they lived in households with better economic conditions. The score was also higher when the women consumed dishes outside the home or ate snacks. However, there were no significant differences based on religion, occupation, the hygiene index of the household or the care for women index. Finally, it is interesting to observe that the women who themselves rated their diets as insufficient in terms of quantity and quality had a lower DDS-22 than women who rated their diets as sufficient.

\section{Relationship between DDS and antbropometric status}

The DDS-9 in tertiles was not associated with any of the anthropometric indices (BMI, MUAC or $\mathrm{BFP}_{\mathrm{C}}$ ) of the women (Table 5). As for the DDS-22, the mean BMI and $\mathrm{BFP}_{\mathrm{C}}$ of the women surveyed increased with an increase in the diversity score, but the differences were not statistically significant. In addition, there were fewer overweight (including obese) women $\left(\mathrm{BMI} \geq 25 \mathrm{~kg} \mathrm{~m}^{-2}\right.$ ) in the low tertile than in the medium and high tertiles of DDS-22, but the difference was not statistically significant. There was also no difference between the percentages of underweight women between the three categories of DDS-22. The same trends were observed even before adjustment for potential confounders.

To complete this analysis, we tried to establish a link between diversity in 'unhealthy' foods and overweight in women. We thus constructed another dietary diversity score based on specific food items rich in lipids and/or carbohydrates: nuts and seeds, fatty meat, vegetable fats, animal fats, frying oil, red palm oil, cheese, sugar and sweetened foods, sweetened drinks and alcohol (1 point for each food group consumed in the previous $24 \mathrm{~h}$ ). We analysed this 'excess score' as a function of the women's anthropometrics, but no association was found.

\section{Discussion}

A major finding in our study was the striking difference between the DDS- 9 and the DDS-22 in terms of their relationships with sociodemographic or economic characteristics. Indeed, the DDS-9 was not associated with the sociodemographic and economic characteristics of the women, except with their perception of their diet in terms of quantity. On the other hand, the DDS based on the list of 22 food groups was associated with almost all of the sociodemographic and economic variables analysed. On the whole, the more privileged women (in terms of education, wealth, care, etc.) were those who had a higher DDS-22. Furthermore, we did not find any association between the DDS-9 and the anthropometric status of the women living in this urban area, whatever the anthropometric indices used. Neither did we find a statistically significant relationship between the DDS-22 and women's anthropometric indices, even though there was a general trend showing that anthropometric indices increased with the DDS-22.

However, other studies conducted in developing countries have demonstrated that a DDS, even calculated from a short list of food groups, was able to discriminate 


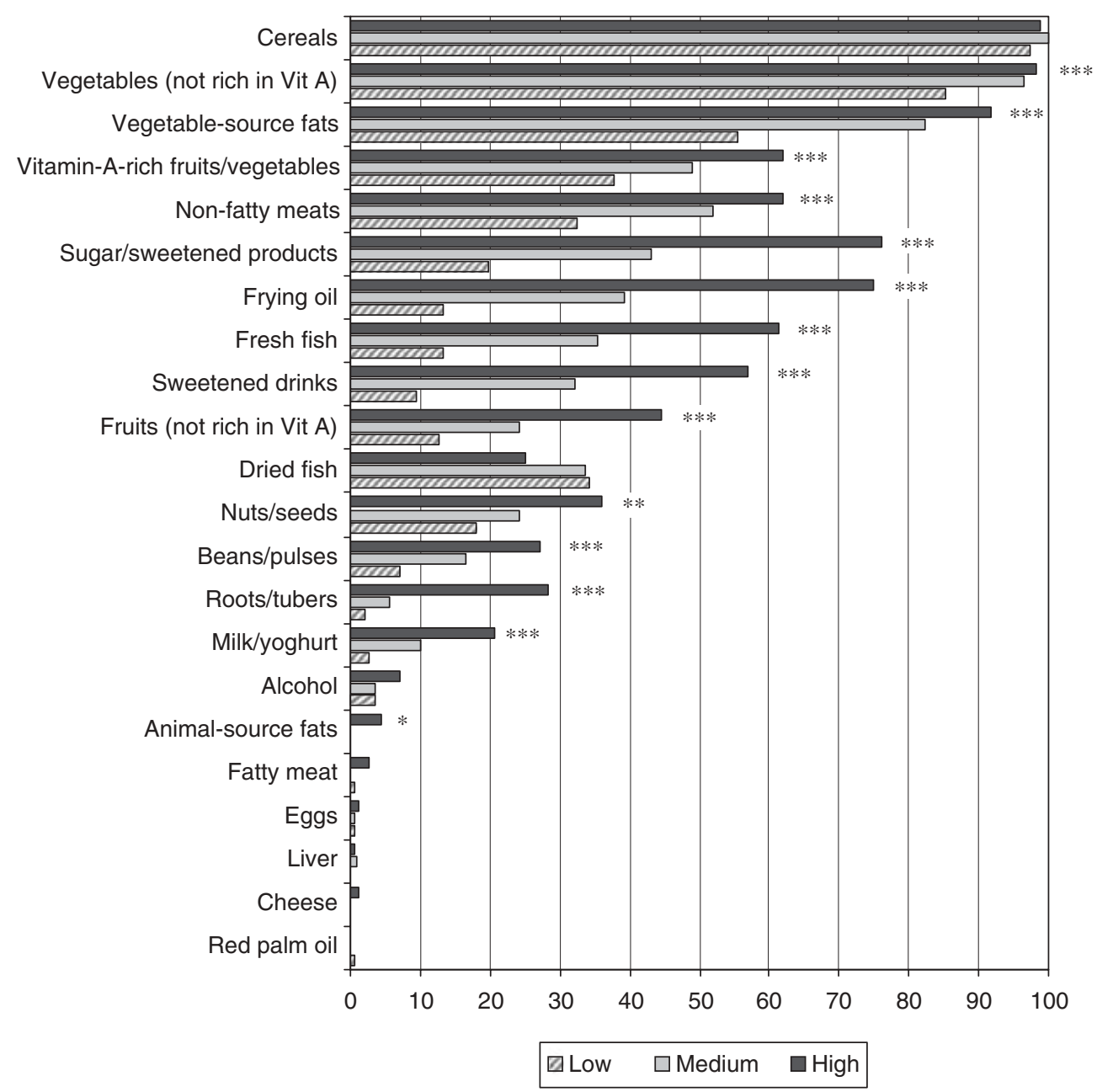

Fig. 2 Percentage of women who consumed food groups as a function of tertiles of the dietary diversity score based on the list of 22 food groups: ${ }^{\star \star \star} P<0.0001 ;{ }^{\star \star} P<0.001 ;{ }^{\star} P<0.01$

Table 3 Frequency of dishes, sauces, snacks and drinks consumed over the 24-h period as a function of dietary diversity scores in tertiles

\begin{tabular}{llclccc}
\hline & & $n$ & Dishes & Sauces & Snacks & Drinks \\
\hline DDS-9 & Low & 180 & 2.4 & 2.1 & 0.4 & 0.5 \\
& Medium & 228 & 2.6 & 2.1 & 0.8 & 0.5 \\
& High & 149 & 2.7 & 1.9 & 1.5 & 0.8 \\
$P$-value & & & 0.0006 & 0.001 & $<0.0001$ & 0.001 \\
DDS-22 & Low & 182 & 2.5 & 2.2 & 0.4 & 0.2 \\
& Medium & 220 & 2.6 & 2.0 & 0.8 & 0.6 \\
& High & 155 & 2.7 & 1.8 & 1.5 & 1.1 \\
$P$-value & & & 0.02 & $<0.0001$ & $<0.0001$ & $<0.0001$ \\
& & & & &
\end{tabular}

DDS-9 - dietary diversity score calculated from the list of nine food groups; DDS-22 - dietary diversity score calculated from the list of 22 food groups.

between individuals or households at least from a socioeconomic standpoint. In Mali, Hatloy et al. demonstrated that a DDS calculated from a list of 10 food groups was related to the socio-economic status of households, but in that study the DDS was calculated at the household level and not at the individual level ${ }^{36}$. Hoddinott and Yohannes also demonstrated that a DDS calculated from a list of 12 food groups was associated with a proxy for household income; but the DDS was also calculated at the household level and, in addition, the analyses included both urban and rural areas in several developing countries ${ }^{10}$. Furthermore, this kind of association was also found with a similar DDS-9 calculated among women living in rural Burkina Faso ${ }^{18}$.

One possible explanation for the lack of such a relationship in the urban context is the huge difference in dietary diversity between urban and rural areas, the DDS-9 being higher in the urban area than in the rural area (respectively $4.9 \pm 1.0$ vs. $3.5 \pm 1.5$ ). Even the women living in the lowest economic conditions in the urban area had a higher DDS-9 $(4.8 \pm 1.0)$ than women living in the rural area. Thus, almost all the women in our urban sample consumed at least four food groups over a 24-h period, whereas this corresponded to a high dietary diversity in the rural area and represented only $36 \%$ of the sample. Access to food was much more varied in the 
Table 4 Relationships between dietary diversity scores and socio-economic characteristics of the women

\begin{tabular}{|c|c|c|c|c|c|}
\hline & $n$ & Mean DDS-9 (SEM) & $P$-value & Mean DDS-22 (SEM) & $P$-value \\
\hline \multicolumn{6}{|l|}{ District } \\
\hline Taabtenga & 246 & $4.9(0.06)$ & \multirow[t]{2}{*}{0.3} & $6.2(0.12)$ & \multirow[t]{2}{*}{0.04} \\
\hline Wemtenga & 235 & $5.0(0.06)$ & & $6.6(0.11)$ & \\
\hline \multicolumn{6}{|l|}{ Age (years) } \\
\hline$<25$ & 112 & $5.1(0.08)$ & \multirow[t]{5}{*}{0.2} & $6.7(0.15)$ & \multirow[t]{5}{*}{0.02} \\
\hline $25-29$ & 126 & $4.9(0.08)$ & & $6.5(0.15)$ & \\
\hline $30-39$ & 140 & $4.9(0.08)$ & & $6.3(0.15)$ & \\
\hline $40-49$ & 61 & $4.8(0.13)$ & & $6.2(0.27)$ & \\
\hline$\geq 50$ & 42 & $4.7(0.16)$ & & $5.8(0.29)$ & \\
\hline \multicolumn{6}{|l|}{ Marital status } \\
\hline Single & 130 & $5.0(0.08)$ & \multirow[t]{3}{*}{0.5} & $6.7(0.14)$ & \multirow[t]{3}{*}{0.01} \\
\hline Married & 310 & $4.9(0.05)$ & & $6.3(0.10)$ & \\
\hline Widowed/divorced & 34 & $4.7(0.19)$ & & $5.8(0.33)$ & \\
\hline \multicolumn{6}{|l|}{ Religion } \\
\hline Moslem & 285 & $4.9(0.05)$ & \multirow[t]{2}{*}{0.5} & $6.4(0.11)$ & \multirow[t]{2}{*}{0.6} \\
\hline Christian & 189 & $4.9(0.07)$ & & $6.3(0.13)$ & \\
\hline \multicolumn{6}{|l|}{ Ever attended school } \\
\hline No & 201 & $4.9(0.07)$ & \multirow[t]{2}{*}{0.7} & $6.1(0.12)$ & \multirow[t]{2}{*}{0.0005} \\
\hline Yes & 280 & $4.9(0.05)$ & & $6.6(0.11)$ & \\
\hline \multicolumn{6}{|l|}{ Occupation } \\
\hline None & 233 & $4.9(0.06)$ & \multirow[t]{3}{*}{0.6} & $6.3(0.12)$ & \multirow{3}{*}{0.3} \\
\hline Yes, in the formal sector (private or public) & 43 & $4.9(0.12)$ & & $6.7(0.22)$ & \\
\hline Yes, in the informal sector & 204 & $4.9(0.07)$ & & $6.4(0.13)$ & \\
\hline Economic index of the household & & & & & \\
\hline Low & 159 & $4.8(0.08)$ & 0.2 & $6.0(0.14)$ & 0.0004 \\
\hline Medium & 161 & $4.9(0.07)$ & & $6.5(0.15)$ & \\
\hline High & 161 & $5.0(0.07)$ & & $6.7(0.13)$ & \\
\hline Monthly expenditure of household for food sto & & & & & \\
\hline 0 (no stock) & 144 & $4.9(0.08)$ & 0.8 & $6.0(0.14)$ & 0.002 \\
\hline $0-10000$ & 72 & $4.9(0.11)$ & & $6.3(0.21)$ & \\
\hline $10000-20000$ & 130 & $4.9(0.08)$ & & $6.4(0.15)$ & \\
\hline $20000-30000$ & 77 & $4.9(0.10)$ & & $6.7(0.22)$ & \\
\hline$>30000$ & 58 & $5.0(0.12)$ & & $7.0(0.25)$ & \\
\hline Hygiene index of the household & & & & & \\
\hline Low & 149 & $4.8(0.07)$ & 0.3 & $6.2(0.15)$ & 0.06 \\
\hline Medium & 211 & $4.9(0.07)$ & & $6.4(0.12)$ & \\
\hline High & 121 & $5.0(0.08)$ & & $6.7(0.16)$ & \\
\hline Care for women index & & & & & \\
\hline Low & 151 & $4.9(0.07)$ & 0.2 & $6.3(0.14)$ & 0.3 \\
\hline Medium & 200 & $5.0(0.07)$ & & $6.5(0.13)$ & \\
\hline High & 130 & $4.8(0.08)$ & & $6.3(0.15)$ & \\
\hline Consumption of meals outside the home & & & & & \\
\hline Never & 158 & $4.8(0.08)$ & 0.08 & $6.0(0.15)$ & 0.002 \\
\hline Rare/occasional & 111 & $5.0(0.10)$ & & $6.6(0.17)$ & \\
\hline Often & 211 & $5.0(0.06)$ & & $6.6(0.11)$ & \\
\hline Snacking & & & & & \\
\hline Never & 23 & $4.6(0.24)$ & 0.4 & $5.9(0.48)$ & 0.01 \\
\hline Rare/occasional & 96 & $4.9(0.09)$ & & $6.0(0.17)$ & \\
\hline Often & 354 & $4.9(0.05)$ & & $6.6(0.09)$ & \\
\hline Perception of the quantity of the diet & & & & & \\
\hline Insufficient & 97 & $4.7(0.10)$ & 0.01 & $5.8(0.18)$ & 0.0003 \\
\hline Sufficient & 378 & $5.0(0.05)$ & & $6.6(0.09)$ & \\
\hline Perception of the quality of the diet & & & & & \\
\hline Not varied enough & 227 & $4.8(0.06)$ & 0.09 & $6.0(0.12)$ & $<0.0001$ \\
\hline Rather varied & 248 & $5.0(0.06)$ & & $6.8(0.11)$ & \\
\hline
\end{tabular}

DDS-9 - dietary diversity score calculated from the list of nine food groups; SEM - standard error of the mean; DDS-22 - dietary diversity score calculated from the list of 22-food groups; FCFA - African Financial Community Franc.

urban area, with more women who consumed vegetables (other than vitamin-A-rich vegetables), fats, meat and fish, fruits (other than vitamin-A-rich fruits) and, to a lesser extent, milk and dairy products. The diversity within these food groups was also higher. This higher diversity was mainly due to higher frequencies of consumption of snacks, drinks and to a lesser extent dishes. On the other hand, we observed that the frequency of consumption of sauces decreased with an increase in the DDS-9 or DDS-22. This could be explained by the type of dishes consumed. We can assume that a woman with a low DDS consumed more basic dishes such as rice or tô (porridge which is the national dish in Burkina Faso), i.e. dishes which are always combined with a sauce, whereas a 
Table 5 Relationships between dietary diversity scores and women's anthropometric status*

\begin{tabular}{|c|c|c|c|c|c|c|c|c|c|}
\hline & & $n$ & $\begin{array}{c}\mathrm{BMl} \\
\left(\mathrm{kg} \mathrm{m}^{-2}\right)\end{array}$ & $\begin{array}{c}\text { MUAC+ } \\
(\mathrm{cm})\end{array}$ & $\begin{array}{l}\mathrm{BFP}_{\mathrm{c}} \neq \\
(\%)\end{array}$ & $\begin{array}{c}\text { Underweight§ } \\
(\%)\end{array}$ & $\begin{array}{c}\text { Normal weight } \\
(\%)\end{array}$ & $\begin{array}{c}\text { Overweight } \| \\
(\%)\end{array}$ & $\begin{array}{c}\text { OR } \\
(95 \% \mathrm{Cl})\end{array}$ \\
\hline \multirow[t]{3}{*}{ DDS-9 } & Low & 159 & 24.1 & 28.7 & 35.6 & 8.5 & 56.0 & 35.5 & 1.00 (referent) \\
\hline & Medium & 199 & 24.3 & 28.9 & 36.0 & 5.4 & 59.5 & 35.0 & $0.93(0.57 ; 1.52)$ \\
\hline & High & 123 & 24.5 & 29.1 & 36.2 & 9.1 & 52.5 & 38.4 & $1.15(0.68 ; 1.97)$ \\
\hline$P$-value & & & 0.8 & 0.7 & 0.8 & & & 0.6 & \\
\hline \multirow[t]{3}{*}{ DDS-22 } & Low & 165 & 23.8 & 28.5 & 35.0 & 7.3 & 61.8 & 30.8 & 1.00 (referent) \\
\hline & Medium & 190 & 24.5 & 29.1 & 36.3 & 6.2 & 54.5 & 39.3 & $1.44(0.87 ; 2.38$ \\
\hline & High & 126 & 24.5 & 29.1 & 36.4 & 9.3 & 52.6 & 38.1 & $1.45(0.85 ; 2.49)$ \\
\hline$P$-value & & & 0.3 & 0.3 & 0.2 & & & 0.5 & \\
\hline
\end{tabular}

$\mathrm{BMI}$ - body mass index; MUAC - mid upper-arm circumference; BFP - body fat percentage corrected; OR - odds ratio; CI - confidence interval; DDS- 9 dietary diversity score calculated from the list of nine food groups; DDS-22 - dietary diversity score calculated from the list of 22 food groups.

${ }^{*}$ Adjusted for age and education of the women, economic and hygiene index of the household, care for women index.

tFor this analysis, $n=470$.

$\ddagger$ For this analysis, $n=458$.

$\S \mathrm{BMl}<18.5 \mathrm{~kg} \mathrm{~m}^{-2}$.

- $18.5 \mathrm{~kg} \mathrm{~m}^{-2} \leq \mathrm{BMl}<25 \mathrm{~kg} \mathrm{~m}^{-2}$

$\| \mathrm{BMI} \geq 25 \mathrm{~kg} \mathrm{~m}^{-2}$.

woman who presented a high DDS may consume more diverse dishes, such as bean dishes, which are not necessarily accompanied by a sauce.

Consequently, the use of a classification system with only a limited number of food groups may have masked some aspects of the dietary diversity of the women in our sample, and thus did not reveal some differences between the socio-economic categories. This assumption is consistent with the fact that there was a correlation between the DDS-22 and the socio-economic status of the women. Indeed, the disaggregation of the foods into 22 food groups enabled discrimination of the women who consumed different types of meat and fish, frying oil in addition to the oil incorporated in dishes, sweetened products, and drinks, i.e. the most privileged women.

The DDS-9 did not enable us to discriminate the women based on their anthropometric status, whereas in a poor rural context we previously found that the women who had a higher DDS-9 were also those with a higher BMI who were less at risk of underweight ${ }^{18}$. In this rural context, we found that higher dietary diversity was associated with higher consumption of energy-dense foods, but as there were no problems of overweight and obesity, this was only reflected in the percentage of underweight women. The DDS-9 thus seems to be more suited to situations in which dietary diversity is very low. Theoretically, the 22 food group classification system would be able to identify women at risk of both micronutrient deficiency and excess in the consumption of 'unhealthy' foods. It is difficult to conclude about current micronutrient deficiencies since we did not make any biological measurements to assess the women's micronutrient status and since these deficiencies have limited consequences for anthropometric status. One could even assume that the interest of disaggregation would probably be limited since we observed that the consumption of liver, red palm oil or other micronutrient-rich foods was in any case very low. On the other hand, we observed that $\mathrm{BMI}$ and $\mathrm{BFP}_{\mathrm{c}}$ were higher when the DDS-22 was higher, even if the DDS-22 did not enable identification of under- or overweight women.

In this study, higher dietary diversity was reflected not only by a higher consumption of 'unhealthy' foods such as sugar and fats, but also by a higher consumption of micronutrients and other healthy foods such as fresh fish, non-fatty meat, and vitamin-A-rich fruits and vegetables. Thus, it is possible that this double-edged diversity makes potential associations between diet and nutritional status difficult to reveal. In Teheran, Azadbakht et al. met a similar problem since these authors found that a higher dietary diversity was associated with higher energy intake and increased obesity in adults, but was also inversely associated with some cardiovascular disease risk factors ${ }^{37}$. In the study in Teheran, a higher DDS was associated with a healthier diet with lower consumption of cholesterol and meat and higher consumption of dietary fibre, fruit, vegetables and vegetable oil. Other studies have reported that more diversified diets might be less healthy from the standpoint of chronic diseases and contribute to the development and maintenance of obesity ${ }^{38-41}$. McCrory et al. also showed that a higher variety of 'unhealthy' foods such as sweets, snacks and carbohydrates was associated with body fatness and that a higher variety of 'healthy' foods such as vegetables was inversely associated with body fatness ${ }^{42}$. In our study we did not find such associations, even using a dietary score based on specific 'unhealthy' items; but in contrast to the previous studies, we did not consider the frequency of consumption or the number of servings consumed. As a consequence, in urban areas, the anthropometric status of individuals probably depends more on the quantity or frequency of foods consumed than on their diversity. In addition, the use of a simple 24-hour dietary recall could explain the absence of associations. However in this study we initially performed three 24-hour dietary recalls on non-consecutive days (two normal days and one day 
off). As the DDS calculated from these repeated recall days led to the same results, we decided to use a single 24-hour recall on a normal day, preferably the first, when women were more likely to give spontaneous answers. Another limitation of our study was that anthropometric indices were the only indicators of the nutritional status of women. As underlined previously, in such a context it would be useful to use other nutritional indicators, such as micronutrient status, to complement anthropometric measurements. Finally, as our sample size was reduced by refusals and missing data, the potential lack of statistical power in the analyses may also explain the absence of any statistical associations.

To conclude, in urban areas, the double-edged aspect of dietary diversity has to be taken into account. Consequently, qualitative measurement of dietary diversity is probably not sufficient to identify populations at risk of under- or overweight. In such a context, the estimation of the quantity of foods consumed, or at least of the usual frequency of consumption, is probably necessary to reach this particular objective. Further research is required to verify this assumption and to continue developing simple and efficient tools that would enable the identification of vulnerable women in urban African contexts.

\section{Acknowledgements}

Sources of funding: The first author received a research allowance from the French Ministry of Research through the Doctoral School 393 of the University of Pierre and Marie Curie (Paris VI). The study was financed by the IRD.

Conflict of interest declaration: The authors have no conflict of interest to declare.

Authorship responsibilities: The study was designed by Y.M.-P., M.S., Hb.D. and F.D. P.D. and M.S. collected the data. M.S., P.T., P.D. and Y.M.-P. analysed the data. M.S. and Y.M.-P. wrote the first draft of the article and then all co-authors contributed to finalise the document.

Acknowledgements: The authors thank the fieldworkers, the ISSP and the population of Wemtenga and Taabtenga for their collaboration, which enabled the successful collection of data.

\section{References}

1 Randall E, Nichaman MZ, Contant CF Jr. Diet diversity and nutrient intake. Journal of the American Dietetic Association 1985; 85(7): 830-6.

2 Krebs-Smith SM, Smiciklas-Wright H, Guthrie HA, KrebsSmith J. The effects of variety in food choices on dietary quality. Journal of the American Dietetic Association 1987; 87(7): 897-903.
3 Drewnowski A, Henderson SA, Driscoll A, Rolls BJ. The Dietary Variety Score: assessing diet quality in healthy young and older adults. Journal of the American Dietetic Association 1997; 97(3): 266-71.

4 Cox DR, Skinner JD, Carruth BR, Moran J 3rd, Houck KS. A Food Variety Index for Toddlers (VIT): development and application. Journal of the American Dietetic Association 1997; 97(12): 1382-6.

5 Guthrie HA, Scheer JC. Validity of a dietary score for assessing nutrient adequacy. Journal of the American Dietetic Association 1981; 78(3): 240-5.

6 Hatloy A, Torheim LE, Oshaug A. Food variety - a good indicator of nutritional adequacy of the diet? A case study from an urban area in Mali, West Africa. European Journal of Clinical Nutrition 1998; 52(12): 891-8.

7 Ogle BM, Hung PH, Tuyet HT. Significance of wild vegetables in micronutrient intakes of women in Vietnam: an analysis of food variety. Asia Pacific Journal of Clinical Nutrition 2001; 10(1): 21-30. Erratum in Asia Pacific Journal of Clinical Nutrition 2001; 10(3): 249.

8 Torheim LE, Ouattara F, Diarra MM, Thiam FD, Barikmo I, Hatloy A, et al. Nutrient adequacy and dietary diversity in rural Mali: association and determinants. European Journal of Clinical Nutrition 2004; 58(4): 594-604.

9 Rose D, Tschirley D. Predicting dietary intakes with simple food recall information: a case study from rural Mozambique. European Journal of Clinical Nutrition 2003; 57(10): 1212-21.

10 Hoddinott J, Yohannes Y. Dietary Diversity as a Household Food Security Indicator. Washington, DC: FANTA Project, Academy for Educational Development, 2002.

11 Aboussaleh Y, Ahami AO, Azzaoui FZ, El Hioui M, Boukhari A. A diversified diet may reduce school age children stunting in North Western Morocco. Asia Pacific Journal of Clinical Nutrition 2004; 13(Suppl.): S116.

12 Arimond M, Ruel MT. Progress in Developing an Infant and Child Feeding Index: An Example using the Ethiopia Demographic and Health Survey 2000. Washington, DC: International Food Policy Research Institute, 2002.

13 Benefice E, Monroy SL, Jimenez S, Lopez R. Nutritional status of Amerindian children from the Beni River (lowland Bolivia) as related to environmental, maternal and dietary factors. Public Health Nutrition 2006; 9(3): 327-35.

14 Sawadogo PS, Martin-Prevel Y, Savy M, Kameli Y, Traissac P, Traore AS, et al. An infant and child feeding index is associated with the nutritional status of 6- to 23-month-old children in rural Burkina Faso. Journal of Nutrition 2006; 136(3): 656-63.

15 Tarini A, Bakari S, Delisle H. [The overall nutritional quality of the diet is reflected in the growth of Nigerian children]. Santé 1999; 9(1): 23-31.

16 Martin-Prevel Y, Savy M, Traissac P, Sawadogo P, Kameli Y, Delpeuch F. Relationship between food variety/diversity scores and nutritional status of adults and adolescents in rural Burkina Faso. Workshop on Dietary Diversity, Dietary Quality and Child Growth, October 11-13. Rome: Food and Agriculture Organization/International Food Policy Research Institute/World Health Organization, 2004.

17 Savy M, Martin-Prevel Y, Sawadogo P, Kameli Y, Delpeuch F. Use of variety/diversity scores for diet quality measurement: relation with nutritional status of women in a rural area in Burkina Faso. European Journal of Clinical Nutrition 2005; 59(5): 703-16.

18 Savy M, Martin-Prevel Y, Traissac P, Delpeuch F. Measuring dietary diversity in rural Burkina Faso: comparison of a 1-day and a 3-day dietary recall. Public Health Nutrition 2007; 10(1): 71-8.

19 Bouzitou GD, Fayomi B, Delisle H. [Child malnutrition and maternal overweight in same households in poor urban areas of Benin]. Santé 2005; 15(4): 263-70. 
20 Doak CM, Adair LS, Bentley M, Monteiro C, Popkin BM. The dual burden household and the nutrition transition paradox. International Journal of Obesity 2005; 29(1): 129-36.

21 World Health Organization (WHO)/Food Agriculture Organization (FAO). Report of the Joint WHO/FAO Expert Consultation on Diet, Nutrition and the Prevention of Chronic Diseases. Geneva: WHO, 2002.

22 Patterson RE, Haines PS, Popkin BM. Diet quality index: capturing a multidimensional behavior. Journal of the American Dietetic Association 1994; 94(1): 57-64.

23 Kennedy E, Ohls J, Carlson S, Fleming K. The Healthy Eating Index: design and applications. Journal of the American Dietetic Association 1995; 95(10): 1103-8.

24 Thiele S, Mensink GB, Beitz R. Determinants of diet quality. Public Health Nutrition 2004; 7(1): 29-37.

25 Basiotis PP, Guthrie JF, Bowman SA, Welsh SO. Construction and evaluation of a Diet Status Index. Family Economics and Nutrition Review 1995; 8(2): 2-13.

26 Hudson G. Food intake in a west African village. Estimation of food intake from a shared bowl. British Journal of Nutrition 1995; 73(4): 551-69.

27 Institut National de la Statistique et de la Démographie (INSD). Analyses des Données du Recensement Général de la Population et de l'Habitation de 1996: Ouagadougou, Burkina Faso. Ouagadougou: INSD, 2000.

28 Institut National de la Statistique et de la Démographie (INSD)/ORC Macro. Enquête Démographique et de Santé du Burkina Faso, 2003. Calverton, MD: INSD/ORC Macro, 2004.

29 Food Agriculture Organization (FAO)/International Food Policy Research Institute (IFPRI)/World Health Organization (WHO). Workshop on Dietary Diversity, Dietary Quality and Child Growth, October 11-13. Rome: FAO/WHO/ IFPRI, 2004

30 World Health Organization (WHO). Physical Status: The Use and Interpretation of Anthropometry. Geneva: WHO, 1995.

31 Gartner A, Dioum A, Maire B, Delpeuch F, Schutz Y. Comparison of foot-to-foot impedance with air displacement plethysmography to evaluate body composition in African women. International Journal of Body Composition Research 2003; 1(4): 137-45.
32 Traissac P, Delpeuch F, Maire B, Martin-Prével Y, Cornu A, Trèche S. [Building a summary index of the households' economic level in nutritional surveys. Application examples in the Congo]. Revue d'Epidémiologie et de Santé Publique 1997; 45(Suppl. 1): 114-15.

33 Lauritsen JM, Bruus M, Myatt M. EpiData, A Tool for Validated Data Entry and Documentation of Data. UK: County of Denmark and Brixton Health, 2000.

34 SAS Institute Inc. The SAS System ${ }^{\mathbb{R}}$, Version 9.1. Cary, NC: SAS Institute Inc., 2004.

35 Gallagher D, Heymsfield SB, Heo M, Jebb SA, Murgatroyd PR, Sakamoto Y. Healthy percentage body fat ranges: an approach for developing guidelines based on body mass index. American Journal of Clinical Nutrition 2000; 72(3): 694-701.

36 Hatloy A, Hallund J, Diarra MM, Oshaug A. Food variety, socioeconomic status and nutritional status in urban and rural areas in Koutiala (Mali). Public Health Nutrition 2000; 3(1): 57-65.

37 Azadbakht L, Mirmiran P, Esmaillzadeh A, Azizi F. Dietary diversity score and cardiovascular risk factors in Tehranian adults. Public Health Nutrition 2005; 9(6): 728-36.

38 Kennedy E. Dietary diversity, diet quality, and body weight regulation. Nutrition Reviews 2004; 62(7): S78-81.

39 Raynor HA, Epstein LH. Dietary variety, energy regulation, and obesity. Psychological Bulletin 2001; 127(3): 325-341.

40 Raynor HA, Jeffery RW, Tate DF, Wing RR. Relationship between changes in food group variety, dietary intake, and weight during obesity treatment. International Journal of Obesity and Related Metabolic Disorders 2004; 28(6): 813-20.

41 Ponce X, Ramirez E, Delisle H. A more diversified diet among Mexican men may also be more atherogenic. Journal of Nutrition 2006; 136(11): 2921-7.

42 McCrory MA, Fuss PJ, McCallum JE, Yao M, Vinken AG, Hays NP, et al. Dietary variety within food groups: association with energy intake and body fatness in men and women. American Journal of Clinical Nutrition 1999; 69(3): $440-7$. 